\title{
Algebraic cycles on the Jacobian of a curve with a linear system of given dimension
}

\author{
Fabien Herbaut \\ Dedicated to Joseph Steenbrink on the occasion of his 60th birthday
}

\begin{abstract}
We present relations between cycles with rational coefficients modulo algebraic equivalence on the Jacobian of a curve. These relations depend on the linear systems that the curve admits. They are obtained in the tautological ring, the smallest subspace containing (an embedding of) the curve and closed under the basic operations of intersection, Pontryagin product and the pullback and pushdown induced by homotheties.
\end{abstract}

\section{Introduction}

In this paper we extend a theorem of Colombo and van Geemen for $d$-gonal curves to linear systems of higher dimension. To express our results in $\S 1.3$ we recall in $\S 1.1$ the Beauville decomposition theorem which enlightens the structure of the tautological ring described in $\S 1.2$. The main points of the proof are summarised in $\S 1.4$.

1.1 We use the notation $C H(X)$ for the Chow ring associated to a smooth algebraic variety $X$, and $A(X)$ for its quotient modulo algebraic equivalence. These rings will always be considered tensored by $\mathbb{Q}$. The brackets $[V]$ mean we consider the class of a subvariety $V$ of $X$. Let $X$ be an Abelian variety over $\mathbb{C}$ whose group law we denote $m$ and dual variety we denote $\hat{X}$. It admits homotheties $x \mapsto k x$ which we will also denote $k$ for $k \in \mathbb{Z}$. We have another product between algebraic cycles, the Pontryagin product, defined between two cycles $\alpha$ and $\beta$ by $\alpha * \beta=m_{*}\left(p^{*} \alpha . q^{*} \beta\right)$ with $p$ and $q$ the two projections associated to the product $X \times X$. Beauville introduced in [Bea83] the Fourier transform $\mathcal{F}$, a $\mathbb{Q}$-linear automorphism between $A(X)$ and $A(\hat{X})$ which exchanges the two products. He used it to prove in [Bea86] the decomposition theorem which states that the operators $k_{*}$ and $k^{*}$ simultaneously diagonalize. For each codimension $p$ the subspace $A^{p}(X)$ splits:

$$
\begin{array}{r}
A^{p}(X)=\bigoplus_{i=p-g}^{g} A_{(i)}^{p}(X) \quad \text { where } \alpha \in A_{(i)}^{p}(X) \text { if and only if for all } k \in \mathbb{Z} \quad k_{*} \alpha=k^{2 g-2 p+i} \alpha \\
\text { (or, equivalently, } k^{*} \alpha=k^{2 p-i} \alpha \text { ). }
\end{array}
$$

It is conjectured that $A_{(i)}^{p}(X)$ vanishes for $i<0$. This is proven if $p \in\{0,1, g-2, g-1, g\}$.

Received 24 May 2006, accepted in final form 14 December 2006.

2000 Mathematics Subject Classification 14C15, 14C20, 14C25, $14 \mathrm{~K} 12$.

Keywords: algebraic cycles, algebraic equivalences, linear systems, Jacobian.

This journal is (C) Foundation Compositio Mathematica 2007. 


\section{F. Herbaut}

1.2 Let $C$ be a smooth projective complex curve of genus $g \geqslant 2$ and $J C$ its Jacobian variety. As $C$ embeds into $J C$, we can consider the associated cycle $[C]$ in $A^{g-1}(J C)$ and its decomposition ${ }^{1}$ :

$$
[C]=C_{(0)}+\cdots+C_{(g-1)} \quad \text { where } C_{(i)} \in A_{(i)}^{g-1}(J C) .
$$

Note that the cycle $[C]$ does not depend on the embedding of the curve, as we work modulo algebraic equivalence. The tautological ring $R$ is the smallest subspace of $A(J C)$ which contains $[C]$ and is stable under the two products and the operators $k_{*}$ and $k^{*}$. Actually, $R$ is finite-dimensional: Beauville proved in [Bea04] that $R$ is the subalgebra for the Pontryagin product generated by $C_{(0)}, \ldots, C_{(g-1)}$. So we can describe $R$ as a quotient of the $\mathbb{Q}$-algebra $\mathbb{Q}\left[C_{(0)}, \ldots, C_{(g-1)}\right]$ by an ideal of relations we have to determine. However, to discuss the vanishing of algebraic cycles is always a difficult problem. Few results are known for $R$. The cycle $C_{(0)}$ is never zero. Neither are $C_{(1)}$ and $C_{(2)}$ for generic curves of respective genera $g \geqslant 3$ and $g \geqslant 11$ as Ceresa [Cer83] and Fakhruddin [Fak96] proved, respectively. More recently, Ikeda [Ike03] proved for each degree $d$ the existence of a smooth plane curve $C$ for which the component $C_{(i)}$ is nonzero in $C H_{(i)}^{g-1}(J C)$ when $i \leqslant d-3$.

On the other hand, one could ask for vanishing results. Polishchuk has presented in [Pol05] an ideal $I_{g}$ of relations which hold for all curves of genus $g$. For nongeneric curves, Colombo and van Geemen proved the main result.

Theorem (Colombo-van Geemen [CV93]). If $C$ is a d-gonal curve then $C_{(i)}=0$ in $A_{(i)}^{g-1}(J C)$ for $i \geqslant d-1$.

1.3 In this paper, we extend this theorem to curves which admit linear systems of higher dimension. More precisely, we state the following result, where we use the abbreviation $g_{d}^{r}$ for linear systems of dimension $r$ and degree $d$.

Theorem 1. Let $C$ be a curve which admits a base point free $g_{d}^{r}$. For each integer $s \geqslant 0$ the following relation holds in $A_{(s)}^{g-r}(J C)$ :

$$
\sum_{\substack{0 \leqslant a_{1}, \ldots, a_{r} \\ a_{1}+\cdots+a_{r}=s}} \beta\left(d, a_{1}+1, \ldots, a_{r}+1\right) C_{\left(a_{1}\right)} * \cdots * C_{\left(a_{r}\right)}=0,
$$

where

$$
\beta\left(d, a_{1}, \ldots, a_{r}\right)=\sum_{i_{1}=1}^{d} \cdots \sum_{i_{r}=1}^{d}(-1)^{i_{1}+\cdots+i_{r}}\left(\begin{array}{c}
d \\
i_{1}+\cdots+i_{r}
\end{array}\right) i_{1}^{a_{1}} \ldots i_{r}{ }^{a_{r}} .
$$

In $\S 7$ we study applications of this theorem to plane and space curves. For almost all genera $g$ there exists a curve with a $g_{d}^{2}$ (respectively, a $g_{d}^{3}$ ) giving new relations. By new relations we mean that they could not be deduced from the $g_{d^{\prime}}^{1}$ that the $g_{d}^{2}$ (respectively, the $g_{d}^{3}$ ) induces and from knowledge of $I_{g}$. We list such relations for $g \leqslant 9$ in the Tables 1 and 2 .

Do we obtain new vanishing statements $C_{(i)}=0$ in $A_{(i)}^{g-1}(J C)$ ? No, because the monomial relations we obtain are $B(r, d, g) C_{(d-2 r+1)}=0$, where for each dimension $r$ the integer $B(r, d, g)$ is the number of $(r-2)$ planes which cut the curve (mapped to $\mathbb{P}^{r}$ by the $\left.g_{d}^{r}\right)$ in $2 r-2$ points. When such a situation arises, we can construct (by projection) a $g_{d-2 r+2}^{1}$. In this case, the monomial relation above does not teach us more than Colombo and van Geemen's theorem. We explain this in $\S 6$.

\footnotetext{
${ }^{1}$ Perhaps it is useful to recall that $C_{(i)}$ is denoted $\pi_{2 g-2-i} C$ in [CV93] and that Polishchuk worked with the classes $p_{i}=\mathcal{F}\left(C_{(i-1)}\right)$ in $[\mathrm{Pol05}]$.
} 


\section{Algebraic CyCles on the JaCobian of A CURVE}

1.4 The first sections are devoted to the proof of Theorem 1. Let $C_{d}$ be the $d$-fold symmetric product of the curve $C$. Choosing an element of $C_{d}$ provides a morphism $u_{d}: C_{d} \longrightarrow J C$. Throughout the whole paper, $\mathcal{G}$ will denote a $g_{d}^{r}$ and $G_{n}$ the truncated linear system of degree $n$; this is the set of divisors of $C_{n}$ we can complete to a divisor of $\mathcal{G}$ :

$$
G_{n}=\left\{D \in C_{n} \mid \exists E \in C_{d-n}, D+E \in \mathcal{G}\right\} \quad \text { for } 1 \leqslant n \leqslant d .
$$

These truncated linear systems may be considered as subvarieties ${ }^{2}$ of the symmetric products $C_{n}$. The scheme structure is described in [ACGH85, paragraph 3 of ch. VIII]. They play an important role in Colombo and van Geemen's proof. We use the notation $\delta_{i_{1}, \ldots, i_{r}}$ for the generalized diagonals $\delta_{i_{1}, \ldots, i_{r}}=\left\{i_{1} x_{1}+\cdots+i_{r} x_{r} \mid x_{i} \in C\right\}$ in the $\left(\sum i_{u}\right)$-fold symmetric product of $C$. The cornerstone of the present note is the following generalization of a formula ${ }^{3}$ they obtained in the case $r=1$, which expresses the algebraic classes $\left[G_{n}\right]$ (and, in particular, $[\mathcal{G}]=\left[G_{d}\right]$ ) as functions of the diagonals.

TheOREM 2. If $C$ admits a base point free $g_{d}^{r}$ then the following equivalences hold in $A^{n-r}\left(C_{n}\right)$ for $r \leqslant n \leqslant d:$

$$
\left[G_{n}\right]=\sum_{1 \leqslant i_{1} \leqslant \cdots \leqslant i_{r}}\left(\begin{array}{c}
d_{r}^{r} \\
n-\sum_{u=1}^{r} i_{u}
\end{array}\right)\left(\prod_{u=1}^{r} \frac{(-1)^{i_{u}-1}}{i_{u}}\right)\left[\delta_{i_{1}, \ldots, i_{r}}+\left(n-\sum_{u=1}^{r} i_{u}\right) o\right]
$$

where we choose a point $o$ of $C$.

We state in $\S 4$ a relation between $\left[G_{r}\right], \ldots,\left[G_{n}\right]$ for all $r \leqslant n \leqslant d$. It enables us to prove Theorem 2 by induction in $\S 5$. In $\S 6$, we note that $u_{d *}\left[G_{d}\right]$ is zero in $A(J C)$, because $G_{d}$ is $\mathcal{G}$ and $u_{d}$ contracts this projective space onto a point in the Jacobian.

Remark. Please note that in [VK07] van der Geer and Kouvidakis gave simpler relations than those of Theorem 1 using the Grothendieck-Riemann-Roch theorem. Don Zagier proved that the two sets of relations are equivalent.

\section{Brief reminder about the Fourier transform and the tautological ring}

The results recalled here are proven in [Bea83] and [Bea86]. We will identify $J C$ and its dual to consider the Fourier transform $\mathcal{F}: A(J C) \rightarrow A(J C)$. It can be defined by the correspondence associated to the exponential of the class of the Poincaré line bundle in $A(J C \times J C)$. As $\mathcal{F} \circ \mathcal{F}=$ $(-1)^{g}(-1)_{*}$, this is an isomorphism. It exchanges the two products, that is

$$
\mathcal{F}(x . y)=(-1)^{g} \mathcal{F}(x) * \mathcal{F}(y) \text { and } \quad \mathcal{F}(x * y)=\mathcal{F} x . \mathcal{F} y .
$$

The decomposition (1) leads to a bigraduation, in the sense that

$$
A_{(i)}^{p}(J C) \cdot A_{(j)}^{q}(J C) \subset A_{(i+j)}^{p+q}(J C) .
$$

The proof of the existence of the decomposition (1) gives

$$
\mathcal{F} A_{(i)}^{p}(J C)=A_{(i)}^{g-p+i}(J C)
$$

\footnotetext{
${ }^{2}$ They have already been used by Kouvidakis in [Kou93] to construct curves in the symmetric product whose Neron Severi classes are known. Izadi has also used the truncated linear systems in [Iza05] to study how curves deform in Abelian varieties.

${ }^{3}$ Proposition 3.4 of [CV93] states that if $\mathcal{G}$ is $g_{d}^{1}$, then we have the equality in $A^{g-1}(J C)$ :
}

$$
u_{n *}\left[G_{n}\right]=\sum_{i=1}^{n} \frac{(-1)^{i-1}}{i}\left(\begin{array}{c}
d \\
n-i
\end{array}\right) i_{*} C
$$




\section{F. Herbaut}

and so the Pontryagin product is also homogeneous of degree $-g$ with respect to the bigraduation

$$
A_{(i)}^{p}(J C) * A_{(j)}^{q}(J C) \subset A_{(i+j)}^{p+q-g}(J C) .
$$

The subspace $R$ is spanned by homogeneous elements for both graduations, so $R$ is bigraded and if we note $R_{(i)}^{p}$ for the intersection $R_{(i)}^{p}=R \cap A_{(i)}^{p}(J C)$, we have

$$
R=\bigoplus_{\substack{0 \leqslant p \leqslant g \\ 0 \leqslant i \leqslant p}} R_{(i)}^{p}
$$

\section{Relations between truncated linear systems}

Let us consider $\mathcal{G}$, a base point free $g_{d}^{r}$. It induces a nondegenerate morphism ${ }^{4} \Phi: C \rightarrow \mathbb{P}^{r}$ and truncated linear systems $G_{n}$. We will also consider for positive integers $r, n$ the subvariety $\mathcal{H}_{\mathbb{P}^{r}}^{n} \subset$ $\left(\mathbb{P}^{r}\right)^{n}$ of $n$-tuples whose components are contained in a hyperplane

$$
\mathcal{H}_{\mathbb{P}^{r}}^{n}=\left\{\left(y_{i}\right) \in\left(\mathbb{P}^{r}\right)^{n} \mid \exists H \in \mathbb{P}^{r *}, \forall i \in\{1, \ldots, n\}, y_{i} \in H\right\} .
$$

Now we introduce the morphisms $\Psi_{\mathcal{P}}: C^{k} \rightarrow C^{n}$ which are defined for integers $0 \leqslant k \leqslant n$ and for $\mathcal{P}$ an ordered $k$-partition of $\{1, \ldots, n\}$ (this is a partition of $\{1, \ldots, n\}$ into $k$ ordered subsets $\left.A_{1}, \ldots, A_{k}\right)$. We set

$$
\begin{aligned}
\Psi_{\left(A_{1}, \ldots, A_{k}\right)}: C^{k} & \longrightarrow C^{n} \\
x & \longmapsto y
\end{aligned}
$$

where $y_{i}=x_{j}$ if and only if $j$ is the unique integer such that $i \in A_{j}$. We also denote by $\sigma_{k}$ the quotient morphism $\sigma_{k}: C^{k} \rightarrow C_{k}$. We can now state the main result of this section.

Proposition 1. We have, for each integer $n \geqslant r$, the equality in $\mathrm{CH}\left(\mathrm{C}^{n}\right)$

$$
\Phi^{n *}\left[\mathcal{H}_{\mathbb{P}^{r}}^{n}\right]=\sum_{k=r}^{n} \frac{1}{k !} \sum_{\substack{\mathcal{P} \text { ordered } \\ k \text {-partition of }\{1, \ldots, n\}}} \Psi_{\mathcal{P}_{*}}\left(\sigma_{k}{ }^{*}\left[G_{k}\right]\right) .
$$

We first state a set theoretic analogue of Proposition 1 in $\S 3.2$. We conclude in $\S 3.3$ where we prove that $\left(\Phi^{n}\right)^{-1} \mathcal{H}_{\mathbb{P}^{r}}^{n}$ is reduced. In $\S 3.1$ we compute the class of $\mathcal{H}_{\mathbb{P}^{r}}^{n}$ in $C H\left(\left(\mathbb{P}^{r}\right)^{n}\right)$.

3.1 One has, by [Ful83, Proposition 8.4], that

$$
C H\left(\mathbb{P}^{r}\right)=\frac{\mathbb{Q}[h]}{\left(h^{r+1}\right)}
$$

where $h$ is the class of a hyperplane of $\mathbb{P}^{r}$. By [Ful83, Proposition 8.3.7] we have

$$
C H\left(\left(\mathbb{P}^{r}\right)^{n}\right) \simeq \frac{\mathbb{Q}\left[h_{1}, \ldots, h_{n}\right]}{\left(h_{1}^{r+1}, \ldots, h_{n}^{r+1}\right)}
$$

where $h_{i}$ is the class of a hyperplane in $\mathbb{P}^{r}$ at the position $i$. Following this isomorphism, we can state the following.

Proposition 2. For integers $0 \leqslant r \leqslant n$ we have, in $C H\left(\left(\mathbb{P}^{r}\right)^{n}\right)$,

$$
\left[\mathcal{H}_{\mathbb{P}^{r}}^{n}\right]=\sum_{\substack{I \subset\{1, \ldots, n\} \\ \# I I=n-r}}\left(\prod_{a \in I} h_{a}\right)
$$

\footnotetext{
${ }^{4}$ This amounts to saying that no hyperplane of $\mathbb{P}^{r}$ contains $\Phi(C)$.
} 


\section{Algebraic CyCles on the Jacobian of A CURVE}

Proof. Let us consider the incidence variety $\mathcal{I}=\left\{(x, H) \in \mathbb{P}^{r} \times \mathbb{P}^{r *} \mid x \in H\right\}$. Let $\mathcal{B}$ be a basis of $\mathbb{C}^{r+1}$, and $\mathcal{B}^{*}$ the dual basis. Such bases induce systems of coordinates on $\mathbb{P}^{r}$ and $\left(\mathbb{P}^{r}\right)^{*}$. If $\left(\left[x_{0}: \ldots: x_{r}\right],\left[a_{0}: \ldots a_{r}\right]\right)$ represents an element of $\mathbb{P}^{r} \times \mathbb{P}^{r *}$, the equation of $\mathcal{I}$ is $\sum a_{i} x_{i}=0$. So $\mathcal{I}$ is a hypersurface of bidegree $(1,1)$ in $\mathbb{P}^{r} \times \mathbb{P}^{r *}$. Now let us consider the variety $I=\left\{\left(x_{1}, \ldots, x_{n}, H\right) \mid\right.$ $\left.x_{i} \in H \forall i \in\{1, \ldots, n\}\right\}$.

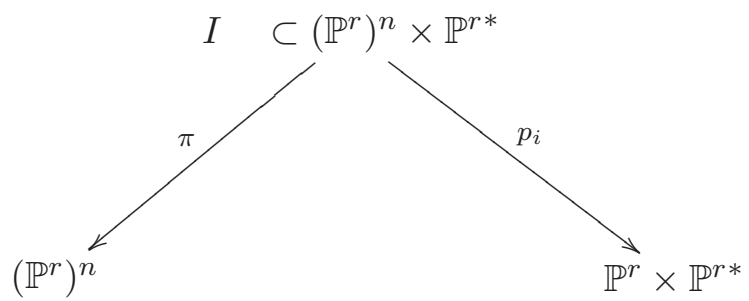

The projection $p_{i}$ maps $\left(x_{1}, \ldots, x_{n}, H\right)$ onto $\left(x_{i}, H\right)$ while $\pi$ maps it onto $\left(x_{1}, \ldots, x_{n}\right)$. We have $\mathcal{H}_{\mathbb{P}^{r}}^{n}=\pi(I)$, and $I$ is the transverse intersection $\bigcap_{i=1}^{n} p_{i}^{-1}(\mathcal{I})$. Following the isomorphism

$$
C H\left(\left(\mathbb{P}^{r}\right)^{n} \times \mathbb{P}^{r *}\right) \simeq \frac{\mathbb{Q}\left[h_{1}, \ldots, h_{n}, h\right]}{\left(h_{1}^{r+1}, \ldots, h_{n}^{r+1}, h^{r+1}\right)}
$$

where $h_{i}$ is the class of a hyperplane at position $i$ and $h$ the class of a hyperplane in $\mathbb{P}^{r *}$, we have in $C H^{n}\left(\left(\mathbb{P}^{r}\right)^{n} \times \mathbb{P}^{r *}\right)$ the equality

$$
[I]=\prod_{i=1}^{n}\left(h_{i}+h\right)
$$

Finally, taking the pushdown $\pi_{*}$ is the same as considering the coefficient of $h^{r}$ in the product (5).

3.2 We establish here the set theoretical equality between cycles leading to Proposition 1.

Proposition 3. For integers $0 \leqslant r \leqslant n$ we have

$$
\left(\Phi^{n}\right)^{-1} \mathcal{H}_{\mathbb{P} r}^{n}=\bigcup_{k=r}^{n}\left(\bigcup_{\mathcal{P}}^{\substack{k-\text { partition } \\ \text { of }\{1, \ldots, n\}}} \Psi_{\mathcal{P}}\left(\sigma_{k}^{-1} G_{k}\right)\right) .
$$

Proof. Let $X$ be an irreducible component of $\left(\Phi^{n}\right)^{-1} \mathcal{H}_{\mathbb{P}^{r}}^{n}$. The generic point $x$ of $X$ is an ordered $n$-tuple with exactly $k$ distinct elements $x_{1}, \ldots, x_{k}$ repeated according to a certain partition $\mathcal{P}$ of $\{1, \ldots, n\}$ into $k$ sets. Then $x$ belongs to $\left(\Phi^{n}\right)^{-1} \mathcal{H}_{\mathbb{P r}}^{n}$ if and only if the points $\Phi\left(x_{i}\right)$ belong to a same hyperplane, that is $\left(x_{1}, \ldots, x_{k}\right) \in \sigma_{k}^{-1} G_{k}$, and then $x \in \Psi_{\mathcal{P}}\left(\sigma_{k}^{-1} G_{k}\right)$. As the other inclusion is straightforward, we have

$$
\left(\Phi^{n}\right)^{-1} \mathcal{H}_{\mathbb{P}^{r}}^{n}=\bigcup_{k=1}^{n} \bigcup_{\substack{\mathcal{P} \\ \text { of }\{1, \ldots, n\}}} \Psi_{\mathcal{P}}\left(\sigma_{k}{ }^{-1} G_{k}\right) .
$$

Suppose that $k \leqslant r$. We know that $k$ points of $\mathbb{P}^{r}$ always lie in a hyperplane, so $G_{k}=C_{k}$ and $\sigma_{k}^{-1} G_{k}=C^{k}$. So

$$
\bigcup_{\begin{array}{c}
\mathcal{P} \text { k-partition } \\
\text { of }\{1, \ldots, n\}
\end{array}} \Psi_{\mathcal{P}}\left(C^{k}\right) \subset \bigcup_{\begin{array}{c}
\mathcal{P} \\
\text { of }\{1, \ldots, n\}
\end{array}} \Psi_{\mathcal{P}}\left(C^{r}\right)
$$

and the proposition follows.

3.3 The following proposition is a sufficient statement for the cycle theoretic application of Proposition 1. 


\section{F. Herbaut}

Proposition 4. The inverse image $\left(\Phi^{n}\right)^{-1} \mathcal{H}_{\mathbb{P}^{r}}^{n}$ is of dimension $r$, and generically reduced along each of its irreducible components of dimension $r$.

We learned the following proof from the referee.

Proof. One knows by Proposition 3 the decomposition of $\left(\Phi^{n}\right)^{-1} \mathcal{H}_{\mathbb{P}^{r}}^{n}$ into irreducible components of dimension $r$. Thus, it suffices to show that at a generic point of each of these components the tangent space to $\left(\Phi^{n}\right)^{-1} \mathcal{H}_{\mathbb{P} r}^{n}$ is $r$-dimensional. Let us denote by $\mathcal{L}$ the line bundle associated to the linear system $\mathcal{G}$ and $W \subset H^{0}(\mathcal{L})$ the space of sections of $\mathcal{L}$. Thus, $\left(\Phi^{n}\right)^{-1} \mathcal{H}_{\mathbb{P}^{r}}^{n}$ is the degeneracy locus of the bundle map

given by evaluation

$$
e v: W \otimes \mathcal{O}_{C^{n}} \longrightarrow \bigoplus_{i=1}^{n} p r_{i}^{*} \mathcal{L}
$$

$$
s \mapsto\left(s\left(x_{1}\right), \ldots, s\left(x_{n}\right)\right)
$$

above a point $x=\left(x_{1}, \ldots, x_{n}\right)$ of $C^{n}$. At a point $x \in C^{n}$ where $e v$ has only a one-dimensional kernel generated by $\sigma$, the tangent space to the degeneracy locus is exactly ker $d \sigma$ where

$$
d \sigma: T_{C^{n}, x}=\bigoplus_{i=1}^{n} T_{C, x_{i}} \longrightarrow \frac{\left(\bigoplus_{i=1}^{n} p r_{i}^{*} \mathcal{L}\right)_{x}}{\operatorname{Im}_{e v_{x}}}
$$

is the projection modulo $I m_{e v_{x}}$ composed with the direct sum of the $d \sigma_{i}: T_{C, x_{i}} \rightarrow \mathcal{L}_{x_{i}}$. Note that all of the differentials $d \sigma_{i}$ are surjective if and only if the section $\sigma$ vanishes transversally at each $x_{i}$. In this case, the tangent space is $r$-dimensional.

To conclude, consider the description of the irreducible components of $\left(\Phi^{n}\right)^{-1} \mathcal{H}_{\mathbb{P} r}^{n}$ given in Proposition 3. As $\Phi$ is nondegenerate, and by Bertini, at the generic point $x$ of each component the kernel ker $e v_{x}$ is generated by one section which vanishes transversally at each $x_{i}$.

\section{Classes of the truncated linear systems}

Let $I, I_{1}, \ldots, I_{r}, J_{1}, \ldots, J_{s}$ be subsets of $\{1, \ldots, n\}$ and let $o_{1}, \ldots, o_{s}$ be points of $C$. We use the notation:

$$
\begin{aligned}
\Delta^{I} & =\left\{\left(x_{1}, \ldots, x_{n}\right) \in C^{n} \mid \forall(i, j) \in I^{2}, x_{i}=x_{j}\right\}, \\
O_{o_{j}}^{I} & =\left\{\left(x_{1}, \ldots, x_{n}\right) \in C^{n} \mid \forall i \in I, x_{i}=o_{j}\right\},
\end{aligned}
$$

and

$$
\Delta^{I_{1}} \ldots \Delta^{I_{r}} O_{o_{1}}^{J_{1}} \ldots O_{o_{s}}^{J_{s}} \quad \text { for the intersection. }
$$

Theorem 3. Assume that the curve $C$ admits a base point free $g_{d}^{r}$ and let $D$ be a reduced divisor of this linear system whose points of the support are pairwise distinct. We have the following equalities in $\mathrm{CH}^{n-r}\left(C^{n}\right)$ and $\mathrm{CH}^{n-r}\left(C_{n}\right)$, respectively:

(i) we have

$$
\sigma_{n}^{*}\left[G_{n}\right]=\sum_{\substack{I_{1}, \ldots, I_{r} \subset\{1, \ldots, n\} \\ o_{1}, \ldots, o_{s} \text { distinct }}}\left(\prod_{u=1}^{r}(-1)^{i_{u}-1}\left(i_{u}-1\right) !\right)\left[\Delta^{I_{1}} \ldots \Delta^{I_{r}} O_{o_{1}}^{\left\{a_{1}\right\}} \ldots O_{o_{s}}^{\left\{a_{s}\right\}}\right]
$$

(ii) we have

$$
\left[G_{n}\right]=\sum_{\substack{1 \leqslant i_{1} \leqslant \cdots \leqslant i_{r} \\ o_{1}, \ldots, o_{s} \text { distinct }}}\left(\prod_{u=1}^{r} \frac{(-1)^{i_{u}-1}}{i_{u}}\right)\left[\delta_{i_{1}, \ldots, i_{r}}+o_{1}+\cdots+o_{s}\right],
$$

where we note that $s=n-\sum_{i=1}^{r} i_{u}$. 


\section{Algebraic CyCles on the Jacobian of A CURVE}

The sum of the first statement is taken over the choice of $r$ subsets $I_{1}, \ldots, I_{r}$ of $\{1, \ldots, n\}$ which are pairwise disjoint. We do not order these sets, except by their growing cardinals $i_{1} \leqslant \cdots \leqslant i_{r}$. For each choice of $I_{1}, \ldots, I_{r}$, we denote by $a_{1}, \ldots, a_{n-\sum i_{u}}$ the elements of $\{1, \ldots, n\} \backslash \bigcup_{k=1}^{r} I_{k}$ we order from the smallest to the largest.

In both statements, the points $o_{1}, \ldots, o_{n-\sum i_{u}}$ are chosen pairwise distinct in the support of the divisor $D$. These points are considered ordered in the sum (i) and unordered in the sum (ii).

Proof. Statement (ii) is a consequence of statement (i) and of the pullback-pushdown formula applied to the degree $n$ ! morphism $\sigma_{n}$ :

$$
\sigma_{n *} \circ \sigma_{n}^{*}=n ! I d_{C H\left(C_{n}\right)} .
$$

To conclude, one counts the diagonals $\Delta^{I_{1}} \ldots \Delta^{I_{r}} O_{o_{1}}^{\left\{a_{1}\right\}} \ldots O_{o_{s}}^{\left\{a_{s}\right\}}$ that $\sigma_{n}$ maps to $\delta_{i_{1}, \ldots, i_{r}}+o_{1}+\cdots+o_{s}$ and the number of antecedents of a generic point $x \in \delta_{i_{1}, \ldots, i_{r}}+o_{1}+\cdots+o_{s}$ by $\sigma_{n}$. We know that $G_{r}=C_{r}$ and $\sigma_{r}^{-1} G_{r}=C^{r}$. We deduce that $\sigma_{r}^{*}\left[G_{r}\right]=\left[C^{r}\right]$. This is Theorem 3 for $n=r$. Suppose that Theorem 3 is proven for $n \leqslant m-1$. By Proposition 1, we have

$$
\sigma_{m}^{*}\left[G_{m}\right]=\Phi^{m *}\left[\mathcal{H}_{\mathbb{P r}}^{m}\right]-\sum_{k=r}^{m-1} \frac{1}{k !} \sum_{\begin{array}{c}
\mathcal{P} \text { ordered } k \text {-partition } \\
\text { of }\{1, \ldots, m\}
\end{array}} \Psi_{\mathcal{P}^{*}}\left(\sigma_{k}{ }^{*}\left[G_{k}\right]\right)
$$

and Proposition 2 gives the class of $\mathcal{H}_{\mathbb{P}^{r}}^{m}$ in $C H\left(\left(\mathbb{P}^{r}\right)^{m}\right)$

$$
\left[\mathcal{H}_{\mathbb{P}^{r}}^{m}\right]=\sum_{\substack{I \subset\{1, \ldots, m\} \\ \# I=m-r}}\left(\prod_{a \in I} h_{a}\right)
$$

By the definition of $D$ we have

$$
\Phi^{m *}\left[\mathcal{H}_{\mathbb{P}^{r}}^{m}\right]=\sum_{\substack{o_{1}, \ldots, o_{m-r} \in \operatorname{Supp}(D) \\ 1 \leqslant a_{1}<\cdots<a_{m-r} \leqslant m}} O_{o_{1}}^{\left\{a_{1}\right\}} \ldots O_{O_{m-r}}^{\left\{a_{m-r}\right\}},
$$

where the points $o_{i}$ are chosen in the support of $D$ and are not necessarily distinct. By the induction hypothesis the classes $\sigma_{k}^{*}\left[G_{k}\right]$ for $k \leqslant m-1$ are sums of classes of varieties $\Delta^{J_{1}} \ldots \Delta^{J_{r}} O_{o_{1}}^{\left\{b_{1}\right\}} \ldots O_{o_{s}}^{\left\{b_{s}\right\}}$ where $\left(J_{1}, \ldots, J_{r},\left\{b_{1}\right\}, \ldots,\left\{b_{s}\right\}\right)$ is a partition of $\{1, \ldots, k\}$. By definition of the morphisms $\Psi_{\mathcal{P}}$, the classes $\Psi_{\mathcal{P}_{*}}\left[\Delta^{J_{1}} \ldots \Delta^{J_{r}} O_{o_{1}}^{\left\{b_{1}\right\}} \ldots O_{o_{s}}^{\left\{b_{s}\right\}}\right]$ are again classes of the form $\left[\Delta^{I_{1}} \ldots \Delta^{I_{r}} O_{o_{1}}^{A_{1}} \ldots O_{O_{s}}^{A_{s}}\right]$ with $\left(I_{1}, \ldots, I_{r}, A_{1}, \ldots, A_{s}\right)$ a partition of $\{1, \ldots, m\}$. So we can express the cycle $\sigma_{m}^{*}\left[G_{m}\right]$ as a linear combination of such classes.

We will fix a partition $\left(I_{1}, \ldots, I_{r}, A_{1}, \ldots, A_{s}\right)$ of $\{1, \ldots, m\}$ and look for which integers $k$ of $\{r, \ldots, m-1\}$ and which partitions $\left(J_{1}, \ldots, J_{r},\left\{b_{1}\right\}, \ldots,\left\{b_{s}\right\}\right)$ of $\{1, \ldots, k\}$ there exists a $k$-ordered partition $\mathcal{P}$ of $\{1, \ldots, m\}$ such that

$$
\Psi_{\mathcal{P}_{*}}\left[\Delta^{J_{1}} \ldots \Delta^{J_{r}} O_{o_{1}}^{\left\{b_{1}\right\}} \ldots O_{o_{s}}^{\left\{b_{s}\right\}}\right]=\Delta^{I_{1}} \ldots \Delta^{I_{r}} O_{o_{1}}^{A_{1}} \ldots O_{o_{s}}^{A_{s}} .
$$

We also have to determine the coefficients associated to these classes in the sum (6).

We will consider the partitions such that for all $u \in\{1, \ldots, r\}, \Psi_{\mathcal{P}}\left(\Delta^{J_{u}}\right)=\Delta^{I_{u}}$. If we denote by $i_{u}$ and $j_{u}$ the cardinals of the sets $I_{u}$ and $J_{u}$, we have by definition of the varieties $\Delta^{I_{u}}$ and the morphisms $\Psi_{\mathcal{P}}$ the inequalities $1 \leqslant j_{u} \leqslant i_{u}$ for all $u \in\{1, \ldots, r\}$. The classes $\Delta^{J_{1}} \ldots \Delta^{J_{r}} O_{o_{1}}^{\left\{b_{1}\right\}} \ldots O_{o_{s}}^{\left\{b_{s}\right\}}$ come from the cycles $\sigma_{k}^{*}\left[G_{k}\right]$ with $k=\sum_{u=1}^{r} j_{u}+s$. All of these contributions are associated to the coefficient

$$
-\frac{1}{k !} \prod_{u=1}^{r}\left(j_{u}-1\right) !(-1)^{j_{u}-1}
$$




\section{F. Herbaut}

which appears in the sum (6). To choose $J_{1}, \ldots, J_{r},\left\{b_{1}\right\}, \ldots,\left\{b_{s}\right\}$ amounts to choosing $r$ disjoint subsets of cardinals $j_{1}, j_{2}, \ldots, j_{r}$. We count $k ! / j_{1} ! \ldots j_{r}$ ! such ways.

To finish we have to choose an ordered partition $\mathcal{P}$ of $\{1, \ldots, m\}$ such that for all $u \in\{1, \ldots, r\}$ we have $\Psi_{\mathcal{P}}\left(\Delta^{J_{u}}\right)=\Delta^{I_{u}}$, and that for each $v \in\{1, \ldots, s\}$ we have $\Psi_{\mathcal{P}}\left(O_{o_{v}}^{\left\{b_{v}\right\}}\right)=O_{o_{v}}^{A_{v}}$. It amounts to choosing ordered partitions of the sets $I_{u}$ in $j_{u}$ subsets. The symbol $\left\{\begin{array}{l}a \\ b\end{array}\right\}$ stands for the Stirling number of the second kind. This is the number of ways to partition a set of $a$ objects into $b$ nonempty sets. ${ }^{5}$ So the number of ways to partition such a set into $b$ ordered sets is $b !\left\{\begin{array}{l}a \\ b\end{array}\right\}$. In this case, we count $\prod_{u=1}^{r} j_{u} !\left\{\begin{array}{c}i_{u} \\ j_{u}\end{array}\right\}$ admissible ways to choose $\mathcal{P}$.

We will distinguish four cases depending on whether the sets $I_{u}$ are singletons (in this case $\Phi^{m *}\left[\mathcal{H}_{\mathbb{P r}}^{m}\right]$ contributes) and whether the sets $A_{v}$ are singletons.

First case: both $I_{u}$ and $A_{v}$ are singletons. Such classes must come from $\Phi^{m *}\left[\mathcal{H}_{\mathbb{P r}}^{m}\right]$, with coefficient 1 by (7). So it is equal to the following product where for all $u \in\{1, \ldots, r\}$ we have $i_{u}=1$ :

$$
\prod_{u=1}^{r}(-1)^{i_{u}-1}\left(i_{u}-1\right) !
$$

Second case: the sets $I_{u}$ are singletons, but one of the sets $A_{v}$ is not. There is, as above, one contribution with coefficient 1 which comes from $\Phi^{m *}\left[\mathcal{H}_{\mathbb{P r}}^{m}\right]$. The cardinals $i_{1}, \ldots, i_{r}$ which are equal to one make the other contributions come from the cycles $\sigma_{r+s}^{*}\left[G_{r+s}\right]$. Precisely, they come from classes $\Delta^{J_{1}} \ldots \Delta^{J_{r}} O_{o_{1}}^{\left\{b_{1}\right\}} \ldots O_{o_{s}}^{\left\{b_{s}\right\}}$ with $j_{1}=\cdots=j_{r}=1$. The coefficient associated is -1 which cancels the contribution of $\Phi^{m *}\left[\mathcal{H}_{\mathbb{P} r}^{m}\right]$.

Third case: one of the sets $I_{u}$ is not a singleton, but the sets $A_{v}$ are singletons. In this case, the pullback $\Phi^{m *}\left[\mathcal{H}_{\mathbb{P}^{r}}^{m}\right]$ does not contribute. On the other hand, we have to enumerate the contributions from the cycles $\sigma_{\sum j_{u}+s}^{*}\left[G_{\sum j_{u}+s}\right]$ for the integers $j_{u}$ which satisfy $1 \leqslant j_{u} \leqslant i_{u}$ and $\sum j_{u}+s<m$. The second inequality is equivalent to $\sum j_{u}<\sum i_{u}$. So the sum is taken over the integers $j_{u}$ which verify $1 \leqslant j_{u} \leqslant i_{u}$ and such that there exists $u \in\{1, \ldots, r\}$ making $j_{u}$ different from $i_{u}$. It is equivalent to consider the sum for all $j_{u}$ such that $1 \leqslant j_{u} \leqslant i_{u}$ and to subtract the contribution corresponding to the term for which $i_{u}=j_{u}$ for each $u \in\{1, \ldots, m\}$. The total contribution is then

$$
-\left(\sum_{j_{1}=1}^{i_{1}} \cdots \sum_{j_{r}=1}^{i_{r}} \prod_{u=1}^{r}(-1)^{j_{u}-1}\left(j_{u}-1\right) !\left\{\begin{array}{c}
i_{u} \\
j_{u}
\end{array}\right\}-\prod_{u=1}^{r}(-1)^{i_{u}-1}\left(i_{u}-1\right) !\right) .
$$

Note that the first sum can be factorized by

$$
\sum_{j_{1}=1}^{i_{1}}(-1)^{j_{1}-1}\left(j_{1}-1\right) !\left\{\begin{array}{l}
i_{1} \\
j_{1}
\end{array}\right\}
$$

which vanishes by Lemma 1 . So the class of $\Delta^{I_{1}} \ldots \Delta^{I_{r}} O_{o_{1}}^{A_{1}} \ldots O_{o_{s}}^{A_{s}}$ again appears with the coefficient

$$
\prod_{u=1}^{r}(-1)^{i_{u}-1}\left(i_{u}-1\right) !
$$

Fourth case: one of the sets $I_{u}$ is not a singleton and one of the sets $A_{v}$ is not a singleton either. In a similar manner, the cycle $\Phi^{m *}\left[\mathcal{H}_{\mathbb{P}^{r}}^{m}\right]$ does not contribute whereas the

\footnotetext{
${ }^{5}$ One could consult [GKP89, ch. 6] to learn more about the Stirling numbers of the second kind.
} 
cycles $\Psi_{\mathcal{P}_{*}}\left[\Delta^{J_{1}} \ldots \Delta^{J_{r}} O_{o_{1}}^{\left\{b_{1}\right\}} \ldots O_{o_{s}}^{\left\{b_{s}\right\}}\right]$ from the pushdown $\sigma_{\sum j_{u}+s}^{*}\left[G_{\sum j_{u}+s}\right]$ contribute as soon as the inequalities $1 \leqslant j_{u} \leqslant i_{u}$ and $\sum j_{u}+s<m$ hold. However, we have $m=\sum i_{u}+\sum \# A_{v}$, and at least one of the cardinals \# $A_{v}$ is strictly greater than one. So the first inequality implies the second, and the class of $\Delta^{I_{1}} \ldots \Delta^{I_{r}} O_{o_{1}}^{A_{1}} \ldots O_{O_{s}}^{A_{s}}$ appears with the coefficient

$$
\sum_{j_{1}=1}^{i_{1}} \cdots \sum_{j_{r}=1}^{i_{r}} \prod_{u=1}^{r}(-1)^{j_{u}-1}\left(j_{u}-1\right) !\left\{\begin{array}{c}
i_{u} \\
j_{u}
\end{array}\right\} .
$$

This concludes the proof of Theorem 3 admitting Lemma 1.

Lemma 1. For an integer $i \geqslant 2$ the following sum vanishes

$$
G(i)=\sum_{j=1}^{i}(-1)^{j-1}(j-1) !\left\{\begin{array}{c}
i \\
j
\end{array}\right\} .
$$

Proof. Use the well-known identity $\left\{\begin{array}{l}i \\ j\end{array}\right\}=j\left\{\begin{array}{l}i-1 \\ j\end{array}\right\}+\left\{\begin{array}{l}i-1 \\ j-1\end{array}\right\}$ (one can consult [GKP89, Table 250]) and thus replace $\left\{\begin{array}{l}i \\ j\end{array}\right\}$ in the definition of $G(i)$.

Now we can simplify Theorem 3 to obtain Theorem 2 . Note that for integers $i_{1}, \ldots, i_{r}$ such that $\sum_{u=1}^{r} i_{u} \leqslant n$ and points of the curve $o, o_{1}, \ldots, o_{n-\sum i_{u}}$, we have

$$
\left[\delta_{i_{1}, \ldots, i_{r}}+o_{1}+\cdots+o_{n-\sum i_{u}}\right]=\left[\delta_{i_{1}, \ldots, i_{r}}+\left(n-\sum i_{u}\right) o\right] \quad \text { in } A^{n-r}\left(C_{n}\right) .
$$

The number of ways to choose $n-\sum i_{u}$ distinct points in the support of a divisor $D \in \mathcal{G}$, that is, among $d$ elements, is $\left(\underset{n-\sum i_{u}}{d}\right)$. Theorem 2 follows.

\section{Relations in the tautological ring modulo algebraic equivalence}

We explain in $\S 5.1$ how to deduce from Theorem 2 the relations of Theorem 1 between the components $C_{(i)}$. In $\S 5.2$ we discuss the nullity of these relations. We use in $\S 5.2$ two lemmas which are proved in $\S 5.3$.

5.1 By definition, all of the divisors of a linear system are linearly equivalent. The morphism $u_{d}$ contracts the $r$-dimensional variety $\mathcal{G}$ into a point of $J C$ so, by definition of the pushdown, we have

$$
u_{d *}[\mathcal{G}]=0 \quad \text { in } C H^{g-r}(J C) .
$$

We have already calculated the class of $\mathcal{G}$ (because $\mathcal{G}=G_{d}$ ) in terms of translates of the generalized diagonals $\delta_{i_{1}, \ldots, i_{r}}+o_{1}+\cdots+o_{s}=\left\{i_{1} x_{1}+\cdots+i_{r} x_{r}+o_{1}+\cdots+o_{s} \mid x_{i} \in C\right\}$. The morphism $u_{d}$ maps these diagonals onto translates of the variety $i_{1} C+\cdots+i_{r} C$ and is one to one. In order to simplify the problem, we will consider relations modulo algebraic equivalence, but one could also express relations modulo the translations in the Chow ring of the Jacobian. From the definition of the Pontryagin product we have $i_{1 *} C * \cdots * i_{r *} C=n\left[i_{1} C+\cdots+i_{r} C\right]$ where $n$ is the degree of the addition morphism.

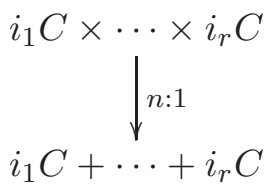




\section{F. Herbaut}

To each sequence $i_{1}, \ldots, i_{r}$ of integers we associate integers $d_{1}, \ldots, d_{u}$ such that

$$
\begin{gathered}
i_{1}=\cdots=i_{d_{1}}, \quad i_{d_{1}} \neq i_{d_{1}+1}, \\
i_{d_{1}+1}=\cdots=i_{d_{1}+d_{2}}, \quad i_{d_{1}+d_{2}} \neq i_{d_{1}+d_{2}+1}, \\
\vdots \\
i_{d_{1}+\cdots+i_{d_{u-1}+1}=\cdots=i_{d_{1}}+\cdots+i_{d_{u}} \text { and } d_{1}+\cdots+d_{u}=r}
\end{gathered}
$$

and we write $\gamma$ for the product

$$
\gamma\left(d, i_{1}, \ldots, i_{r}\right)=d_{1} ! \ldots d_{u} !
$$

The number of antecedents of a generic point of $i_{1} C+\cdots+i_{r} C$ is $d_{1} ! \ldots d_{u}$ !, so we have

$$
i_{1 *} C * \cdots * i_{r *} C=d_{1} ! \ldots d_{u} !\left[i_{1} C+\cdots+i_{r} C\right] .
$$

By (8), Theorem 2, and (9), we obtain

$$
\sum_{1 \leqslant i_{1} \leqslant \cdots \leqslant i_{r} \leqslant n} \frac{(-1)^{i_{1}+\cdots+i_{r}}}{i_{1} \ldots i_{r}}\left(\begin{array}{c}
d \\
i_{1}+\cdots+i_{r}
\end{array}\right) \frac{1}{d_{1} ! \ldots d_{u} !} i_{1 *} C * \cdots * i_{r *} C=0 .
$$

For all integers $i_{1}, \ldots, i_{r}$ such that $1 \leqslant i_{1} \leqslant \cdots \leqslant i_{r}$ we count $\left(\begin{array}{c}r \\ d_{1}, \ldots, d_{u}\end{array}\right)$ different $r$-tuples $\left(j_{1}, \ldots, j_{r}\right)$ we obtain by permutations of the $i_{k}$. So we can write

$$
\sum_{i_{1}=1}^{d} \cdots \sum_{i_{r}=1}^{d} \frac{(-1)^{i_{1}+\cdots+i_{r}}}{i_{1} \ldots i_{r}}\left(\begin{array}{c}
d \\
i_{1}+\cdots+i_{r}
\end{array}\right) i_{1 *} C * \cdots * i_{r *} C=0 .
$$

Now we use the decomposition (2), the action of the operator $i_{*}$ onto the components (that is $\left.i_{*} C_{(a)}=i^{a+2} C_{(a)}\right)$, and multilinearity to write

$$
i_{1 *} C * \cdots * i_{r *} C=\sum_{a_{1}=1}^{g} \ldots \sum_{a_{r}=1}^{g} i_{1}^{a_{1}+2} \ldots i_{r}{ }^{a_{r}+2} C_{\left(a_{1}\right)} * \cdots * C_{\left(a_{r}\right)} .
$$

Then we use the inclusions $A_{(s)}^{p}(J C) * A_{(t)}^{q}(J C) \subset A_{(s+t)}^{p+q-g}(J C)$ and we project (10) onto $A_{(s)}^{g-r}(J C)$ for all $s \geqslant 0$ to prove Theorem 1 .

5.2 The relations we quote are obtained in $A_{(s)}^{g-r}(J C)$. For small values of $s$, these relations are trivial. For example, for $s=0$, the relation is monomial with $C_{(0)}^{* r}$. It should be trivial, otherwise we would obtain the vanishing of a small power of the theta divisor. The first nontrivial relation is obtained for $s=d-r+1$ as stated in the following proposition.

Proposition 5. We have

$$
\beta\left(d, a_{1}, \ldots, a_{r}\right)= \begin{cases}0 & \text { if } a_{1}+\cdots+a_{r}<d-r+1 \\ (-1)^{d} a_{1} ! \ldots a_{r} ! & \text { if } a_{1}+\cdots+a_{r}=d-r+1 .\end{cases}
$$

Proof. We denote $t=i_{1}+\cdots+i_{r}$ and expand $i_{r}^{a_{r}}=\left(t-i_{1}-\cdots-i_{r-1}\right)^{a_{r}}$ using Newton's multinomial formula to get

$$
\begin{aligned}
\beta\left(d, a_{1}, \ldots, a_{r}\right)= & \sum_{t \geqslant 0}(-1)^{t}\left(\begin{array}{l}
d \\
t
\end{array}\right) \sum_{\substack{b, b_{1}, \ldots, b_{r-1} \\
b+b_{1}+\cdots+b_{r-1}=a_{r}}}(-1)^{b_{1}+\cdots+b_{r-1}}\left(\begin{array}{c}
a_{r} \\
b, b_{1}, \ldots, b_{r-1}
\end{array}\right) \\
& \times t^{b} \sum_{i_{1}=0}^{t} \sum_{i_{2}=0}^{t-i_{1}} \cdots \sum_{i_{r-1}=0}^{t-i_{1}-\cdots-i_{r-2}} i_{1}^{a_{1}+b_{1}} \ldots i_{r-1} a^{a_{r-1}+b_{r-1}} .
\end{aligned}
$$




\section{Algebraic CyCles on the Jacobian of A CURVE}

By Lemma 2 below, the lower part of the sum above is a polynomial of degree

$$
\sum_{i=1}^{r-1} a_{i}+\sum_{i=1}^{r-1} b_{i}+b+r-1=\sum_{i=1}^{r} a_{i}+r-1
$$

in $t$ whose leading coefficient is

$$
\frac{\prod_{i=1}^{r-1}\left(a_{i}+b_{i}\right) !}{\left(\sum_{i=1}^{r-1} a_{i}+\sum_{i=1}^{r-1} b_{i}+r-1\right) !}
$$

The equality $\sum_{t=0}^{d}(-1)^{t}\left(\begin{array}{l}d \\ t\end{array}\right) t^{a}=d !\left\{\begin{array}{l}a \\ d\end{array}\right\}(-1)^{d}$ is the result (6.19) in [GKP89]. However, the Stirling number $\left\{\begin{array}{l}a \\ d\end{array}\right\}$ vanishes for $a<d$ and equals one when $a=d$. So when $\sum_{i=1}^{r} a_{i}<d-r+1$, the sum $\beta\left(d, a_{1}, \ldots, a_{r}\right)$ vanishes. In the case of equality $\sum_{i=1}^{r} a_{i}=d-r+1$, it remains to compute the sum

$$
(-1)^{d} d ! \sum_{\substack{b, b_{1}, \ldots, b_{r-1} \\
b+b_{1}+\cdots+b_{r-1}=a_{r}}}\left(\begin{array}{c}
a_{r} \\
b, b_{1}, \ldots, b_{r-1}
\end{array}\right)(-1)^{b_{1}+\cdots+b_{r-1}} \frac{\prod_{i=1}^{r-1}\left(a_{i}+b_{i}\right) !}{\left(\sum_{i=1}^{r-1} a_{i}+\sum_{i=1}^{r-1} b_{i}+r-1\right) !}
$$

which is $(-1)^{d} \prod_{i=1}^{r} a_{i}$ ! by Lemma 3 below.

5.3 We now provide the lemmas used in $\S 5.2$.

Lemma 2. For all positive integers $a_{1}, a_{2}, \ldots, a_{n}$ the sum

$$
\sum_{\substack{0 \leqslant i_{1}, \ldots, i_{n} \\ i_{1}+\cdots+i_{n} \leqslant t}} i_{1}^{a_{1}} \ldots i_{n}^{a_{n}}
$$

is a polynomial of degree $a_{1}+\cdots+a_{n}+n$ in $t$. Its leading term is

$$
\frac{\prod_{i=1}^{n} a_{i} !}{\left(n+\sum_{i=1}^{n} a_{i}\right) !} \text {. }
$$

Proof. Suppose that Lemma 2 is proven up to rank $n$, and write the sum above with $n+1$ terms in this way:

$$
\sum_{i_{1}=0}^{t} i_{1}{ }^{a_{1}}\left(\sum_{\substack{0 \leqslant i_{2}, \ldots, i_{n+1} \\ i_{2}+\cdots+i_{n+1} \leqslant t-i_{1}}} i_{2}^{a_{2}} \ldots i_{n+1}{ }^{a_{n+1}}\right) .
$$

By the induction hypothesis, the term between parentheses is a degree $\left(a_{2}+\cdots+a_{n+1}+n\right)$ polynomial in $t-i_{1}$. Its leading coefficient is $a_{2} ! \ldots a_{n+1} ! /\left(a_{2}+\cdots+a_{n+1}+n\right) !$. We denote it by $P$. There exists a polynomial $Q$ whose degree is less than $a_{2}+\cdots+a_{n+1}+n$ and such that

$$
P(t)=\frac{a_{2} ! \ldots a_{n+1} !}{\left(a_{2}+\cdots+a_{n+1}+n\right) !} t^{a_{2}+\cdots+a_{n+1}+n}+Q(t) .
$$

So (11) is equal to

$$
\frac{a_{2} ! \ldots a_{n+1} !}{\left(a_{2}+\cdots+a_{n+1}+n\right) !} \sum_{i_{1}=0}^{t} i_{1}^{a_{1}}\left(t-i_{1}\right)^{a_{2}+\cdots+a_{n+1}+n}+\sum_{i_{1}=0}^{t} Q\left(t-i_{1}\right) i_{1}^{a_{1}} .
$$

We note $N=a_{2}+\cdots+a_{n+1}+n$ for brevity and develop $\left(t-i_{1}\right)^{N}$ using Newton's binomial formula. The first sum of expression (12) becomes

$$
\sum_{k=0}^{N}(-1)^{k} t^{N-k}\left(\begin{array}{l}
N \\
k
\end{array}\right)\left(\sum_{i_{1}=0}^{t} i_{1}^{a_{1}+k}\right) .
$$




\section{F. Herbaut}

Now we use the induction hypothesis when $n=1$ to prove that the sum between parentheses in (13) is a degree $a_{1}+k+1$ polynomial in $t$ whose leading term is $1 /\left(a_{1}+k+1\right)$. So (13) is a polynomial of degree $a_{1}+N+1$ in $t$ whose leading term is

$$
\sum_{k=0}^{N}(-1)^{k}\left(\begin{array}{l}
N \\
k
\end{array}\right) \frac{1}{a_{1}+k+1}
$$

The simplification of this last sum given by formula (5.51) in [GKP89] enables us to conclude.

In $\S 5.2$ we have used the following equality.

Lemma 3. For $r$ positive integers $a_{1}, a_{2}, \ldots, a_{r}$, we have

$$
\sum_{\substack{0 \leqslant b, b_{1}, \ldots, b_{r-1} \\
b+b_{1}+\cdots+b_{r-1}=a_{r}}}\left(\begin{array}{c}
a_{r} \\
b, b_{1}, \ldots, b_{r-1}
\end{array}\right)(-1)^{b_{1}+\cdots+b_{r-1}} \frac{\prod_{i=1}^{r-1}\left(a_{i}+b_{i}\right) !}{\left(\sum_{i=1}^{r-1} a_{i}+\sum_{i=1}^{r-1} b_{i}+r-1\right) !}=\frac{\prod_{i=1}^{r} a_{i} !}{\left(\sum_{i=1}^{r} a_{i}+r-1\right) !} .
$$

Proof. Write the sum in the following way

$$
\sum_{\substack{b, b_{1}, \ldots, b_{r-1} \\ b+b_{1}+\cdots+b_{r-1}=a_{r}}} \prod_{i=1}^{r-1}\left((-1)^{b_{i}} \frac{\left(a_{i}+b_{i}\right) !}{b_{i} !}\right)\left(\frac{1}{b !\left(\sum_{i=1}^{r} a_{i}+r-1-b\right) !}\right) .
$$

One could consult [GKP89, p. 321] for the following equalities which hold for all $i \in\{1, \ldots, r-1\}$ :

$$
\sum_{b_{i} \geqslant 0} \frac{(-1)^{b_{i}}\left(a_{i}+b_{i}\right) !}{b_{i} !} X^{b_{i}}=a_{i} ! \sum_{b_{i} \geqslant 0}\left(\begin{array}{c}
a_{i}+b_{i} \\
b_{i}
\end{array}\right)(-X)^{b_{i}}=\frac{a_{i} !}{(1+X)^{a_{i}+1}}
$$

We also note that

$$
\begin{aligned}
\sum_{b \geqslant 0} \frac{X^{b}}{b !\left(\sum_{i=1}^{r} a_{i}+(r-1)-b\right) !} & =\frac{\sum_{b \geqslant 0}\left(\begin{array}{c}
\sum_{i=1}^{r} a_{i}+r-1 \\
b
\end{array}\right) X^{b}}{\left(\sum_{i=1}^{r} a_{i}+r-1\right) !} \\
& =\frac{(1+X)^{\sum_{i=1}^{r} a_{i}+r-1}}{\left(\sum_{i=1}^{r} a_{i}+r-1\right) !}
\end{aligned}
$$

so the sum we study is the product of $\prod_{i=1}^{r} a_{i} ! /\left(\sum_{i=1}^{r} a_{i}+r-1\right) !$ by the coefficient of $X^{a_{r}}$ in the formal series

$$
\frac{1}{(1+X)^{a_{1}+1}} \cdots \frac{1}{(1+X)^{a_{r-1}+1}}(1+X)^{a_{1}+\cdots+a_{r}+r-1} .
$$

This is the coefficient of $X^{a_{r}}$ in $(1+X)^{a_{r}}$, so it is 1 .

\section{Linear systems and $(2 r-2)$-secant $r-2$ planes}

The goal of this section is to analyze the relations $C_{(i)}=0$ we can deduce from Theorem 1 . First of all, for $r=1$, one recovers Colombo and van Geemen's theorem. The study of relations when $r \geqslant 2$ makes the following coefficients appear:

$$
A(r, d, g)=\sum_{i=0}^{r-1} \frac{(-1)^{i}}{d-2 r+2}\left(\begin{array}{c}
i+g+r-d-2 \\
i
\end{array}\right)\left(\begin{array}{c}
d-2 r \\
r-1-i
\end{array}\right)\left(\begin{array}{c}
d-r+1-i \\
r-i
\end{array}\right) .
$$

Using the relations of Theorem 1 we will prove the following.

THEOREM 4. If $C$ admits a base point free $g_{d}^{r}$ and if $A(r, d, g) \neq 0$, then we have

$$
C_{(i)}=0 \quad \text { in } A_{(i)}^{g-1} \text { for } i \geqslant d-2 r+1 .
$$




\section{Algebraic Cycles on the Jacobian of a Curve}

However, we will explain how Theorem 4 can also be deduced from the theorem of Colombo and van Geemen because $A(r, d, g)$ is the number of projective spaces of dimension $r-2$ which cut the curve in $2 r-2$ points. So Theorem 4 does not give us new relations, but it is interesting to recover a number of geometrical significance such as the Castelnuovo number from the relations of Theorem 1. It could also suggest the existence of a partial converse to the theorem of Colombo and van Geemen.

\subsection{The case of a $g_{d}^{1}$}

If $C$ admits a base point free $g_{d}^{1}$ then Theorem 1 gives, for $0 \leqslant a \leqslant g-1$,

$$
\beta(d, a+1) C_{(a)}=0 \quad \text { in } A_{(a)}^{g-1} .
$$

The coefficients $\beta(d, a)$ can be expressed in terms of the Stirling numbers of the second kind. One can consult formula (6.19) of [GKP89]:

$$
\beta(d, a)=\sum_{i=1}^{d}(-1)^{i}\left(\begin{array}{l}
d \\
i
\end{array}\right) i^{a}=d !\left\{\begin{array}{l}
a \\
d
\end{array}\right\}(-1)^{d} .
$$

Lastly, $\left\{\begin{array}{c}a+1 \\ d\end{array}\right\}$ is nonzero if and only if $0 \leqslant d \leqslant a+1$, so we have $C_{(a)}=0$ for $a \geqslant d-1$. This is Colombo and van Geemen's result.

\subsection{Proof of Theorem 4}

Proof. By Proposition 5, the first nontrivial relation is obtained in $A_{(d-2 r+1)}^{g-d+2 r-1}(J C)$ :

$$
\sum_{\substack{1 \leqslant a_{1} \leqslant \cdots \leqslant a_{r} \\ a_{1}+\cdots+a_{r}=d-2 r+1}} \frac{\left(a_{1}+1\right) ! \ldots\left(a_{r}+1\right) !}{\gamma\left(a_{1}, \ldots, a_{r}\right)} C_{\left(a_{1}\right)} * \cdots * C_{\left(a_{r}\right)} .
$$

Now multiply (14) by $C_{(0)}^{*(g+r-d-2)}$ and order the terms with respect to the powers of $C_{(0)}$. We introduce $t$ which is the number of integers $a_{i}$ which are equal to zero. So we have $a_{1}=\cdots=a_{t}=0$ and $a_{t+1}>0$. By definition of $\gamma$ we have $\gamma\left(a_{1}, \ldots, a_{r}\right)=t ! \gamma\left(a_{t+1}, \ldots, a_{r}\right)$, and

$$
\sum_{t=0}^{r-1} \sum_{\substack{1 \leqslant a_{t+1} \leqslant \cdots \leqslant a_{r} \\ a_{t+1}+\cdots+a_{r}=d-2 r+1}} \frac{\left(a_{t+1}+1\right) ! \ldots\left(a_{r}+1\right) !}{t ! \gamma\left(a_{t+1}, \ldots, a_{r}\right)} C_{(0)}^{*(t+g+r-2-d)} * C_{\left(a_{t+1}\right)} * \cdots * C_{\left(a_{r}\right)}=0 .
$$

By Fourier transform, $R_{(d-2 r+1)}^{g-1}$ is isomorphic to $R_{(d-2 r+1)}^{d-2 r+2}$. This subspace is spanned by $C_{(d-2 r+1)}$, so its dimension is bounded by 1 . The relations Polishchuk obtained enable us to express $C_{(0)}^{*(t+g+r-2-d)} * C_{\left(a_{t+1}\right)} * \cdots * C_{\left(a_{r}\right)}$ in terms of $C_{(0)}^{*(g+2 r-d-3)} * C_{(d-2 r+1)}$. The corollary of Lemma 0.3 in $[\mathrm{Pol05}]$ gives

$$
\begin{aligned}
& C_{(0)}^{*\left(g-1-\sum_{i=1}^{k} n_{i}\right)} * C_{\left(n_{1}\right)} * \cdots * C_{\left(n_{k}\right)} \\
& \quad=\left((-1)^{k-1} \frac{\left(g-1-\sum_{i=1}^{k} n_{i}\right) !}{\left(g+k-2-\sum_{i=1}^{k} n_{i}\right) !} \frac{\left(\sum_{i=1}^{k} n_{i}\right) !}{n_{1} ! \ldots n_{k} !}\right) C_{(0)}^{*\left(g+k-2-\sum_{i=1}^{k} n_{i}\right)} * C_{\left(1-k+\sum_{i=1}^{k} n_{i}\right)} .
\end{aligned}
$$

Thus (15) becomes a monomial relation whose coefficient is

$$
\sum_{t=0}^{r-1}(-1)^{r+t} \frac{(t+g+r-d-2) !(d-r+1-t) !}{t !(r-t) !}\left(\sum_{\substack{1 \leqslant a_{t+1} \leqslant \cdots \leqslant a_{r} \\ a_{t+1}+\cdots+a_{r}=d-2 r+1}} \frac{(r-t) !}{\gamma\left(a_{t+1}, \ldots, a_{r}\right)}\right) .
$$




\section{F. Herbaut}

We note that $\gamma\left(a_{t+1}, \ldots, a_{r}\right)=\gamma\left(a_{t+1}-1, \ldots, a_{r}-1\right)$ and reindex the sum to obtain

$$
\sum_{\substack{1 \leqslant a_{t+1} \leqslant \cdots \leqslant a_{r} \\
a_{t+1}+\cdots+a_{r}=d-2 r+1}} \frac{(r-t) !}{\gamma\left(a_{t+1}, \ldots, a_{r}\right)}=\sum_{\begin{array}{c}
0 \leqslant b_{1} \leqslant \cdots \leqslant b_{r-t} \\
b_{1}+\cdots+b_{r-t}=d-3 r+1+t
\end{array}} \frac{(r-t) !}{\gamma\left(b_{1}, \ldots, b_{r-t}\right)} .
$$

As $(r-t) ! / \gamma\left(b_{1}, \ldots, b_{r-t}\right)$ is the number of $(r-t)$-tuples we can associate to the integers $\left\{b_{1}\right\}$, $\left\{b_{2}\right\}, \ldots,\left\{b_{r-t}\right\}$, the sum corresponds to the number of positive integers whose sum is $d-3 r+1+t$. If we consider the formal series $1 /(1-X)=1+X+X^{2}+\cdots$, the integer we are looking for appears as the coefficient of $X^{d-3 r+1+t}$ in $(1 /(1-X))^{r-t}$, that is $\left(\begin{array}{c}d-2 r \\ d-3 r+1+t\end{array}\right)$. Now factor (16) by $(g+r-d-2) !(d-2 r+2)$ ! to make the coefficient $A(r, d, g)$ above appear. Note that $C_{(d-2 r+1)}$ is (up to a coefficient) the Fourier transform of $C_{(0)}^{*(g+2 r-d-3)} * C_{(d-2 r+1)}$, so when $A(r, d, g) \neq 0$, we have $C_{(d-2 r+1)}=0$ modulo algebraic equivalence. This concludes the proof of Theorem 4 admitting Lemma 4 below.

The following lemma is a consequence of the relations obtained by Polishchuk. Marini has also noted and proven it in [Mar05].

Lemma 4. Let $i$ be a positive integer. If $C_{(i)}=0$ in $A_{(i)}^{g-1}$, then $C_{(i+1)}=0$ in $A_{(i+1)}^{g-1}$.

Proof. If $g \in\{2,3\}$, the only components which arise are $C_{(0)}$ and $C_{(1)}$, and the cycle $C_{(0)}$ never vanishes because $-\mathcal{F}\left(C_{(0)}\right)$ is the class of the theta divisor (see [Bea04]). Now suppose that $g \geqslant 4$. As the gonality of the curve is less than $g$, apply the theorem of Colombo and van Geemen to obtain $C_{(g-2)}=0$ and the implication $C_{(g-3)}=0 \Rightarrow C_{(g-2)}=0$. Now suppose that $i \leqslant g-4$. The point is that $R_{(i+1)}^{g-1}$, which is spanned by $C_{(i+1)}$, is isomorphic to $R_{(i)}^{i+1}$, which is spanned by $C_{(0)}^{*(g-i-2)} * C_{(i+1)}$ by [Pol05, Corollary 0.3]. Now consider the cycle $C_{(0)} * C_{(1)} * C_{(i)}$ which is proportional to $C_{(0)}^{*(g-i-2)} * C_{(i+1)}$ by the same corollary.

\subsection{The Castelnuovo number}

The Grassmannian $\mathbb{G}(r-1, r+1)$ which parametrises the $(r-2)$-dimensional linear subspaces of $\mathbb{P}^{r}$ is a $(2 r-2)$-fold. The subspaces containing a point of the curve correspond to a codimension one subvariety of $\mathbb{G}(r-1, r+1)$. So one can expect to find a finite number of $\mathbb{P}^{r-2}$ which contains $2 r-2$ points of the curve. Actually, the formula of Castelnuovo computes this number when it is finite:

$$
B(r, d, g)=\sum_{i=0}^{r-1} \frac{(-1)^{i}}{r-i}\left(\begin{array}{c}
d-r-i+1 \\
r-1-i
\end{array}\right)\left(\begin{array}{c}
d-r-i \\
r-1-i
\end{array}\right)\left(\begin{array}{l}
g \\
i
\end{array}\right) .
$$

One could consult [ELMS89] for a proof. When $B(r, d, g)$ is nonzero, consider $2 r-2$ such points and then the hyperplanes which contain these points. The linear system we obtain in this way is a $g_{d-2 r+2}^{1}$ and we can apply the theorem of Colombo and van Geemen. So when $B(r, d, g) \neq 0$, we have $C_{(i)}=0$ in $A_{(i)}^{g-1}$ for $i \geqslant d-2 r+1$. We conclude this second proof of Theorem 4 with the following lemma.

Lemma 5. For each integer $r \geqslant 1$, we have the equality in $\mathbb{Q}[d, g]$ :

$$
A(r, d, g)=B(r, d, g) .
$$

We use the algorithm of Zeilberger to prove that $A(r, d, g)=B(r, d, g)$. We apply it thanks to the software Mathematica and the package ZW that Paule and Schorne have developed [PS95]. It enables us to compute a linear recurrence relation

$$
Q_{2}(r, d, g) X(r+2, d, g)+Q_{1}(r, d, g) X(r+1, d, g)+Q_{0}(r, d, g) X(r, d, g)=0
$$




\section{Algebraic CyCles on the JaCobian of A CURVE}

TABLE 1. Relations deduced from Theorem 1 for plane curves of genus $g \leqslant 9$.

\begin{tabular}{|c|c|c|c|c|}
\hline Genus & $g_{d}^{2}$ & $\begin{array}{l}g_{d^{\prime}}^{1} \text { deduced } \\
\text { from the } g_{d}^{2}\end{array}$ & $\begin{array}{l}\text { Relations deduced from the } g_{d}^{2} \\
\text { with Theorem } 1\end{array}$ & Consequences \\
\hline$g=5$ & $g_{5}^{2}$ & $g_{3}^{1}$ & $3 C_{(0)} * C_{(2)}+C_{(1)}^{* 2}=0$ & \\
\hline$g=6$ & $g_{5}^{2}$ & $g_{4}^{1}$ & $3 C_{(0)} C_{(2)}+C_{(1)}^{2}=0$ & \\
\hline$g=7$ & $g_{6}^{2}$ & $g_{4}^{1}$ & $2 C_{(0)} * C_{(3)}+C_{(1)} * C_{(2)}=0$ & \\
\hline \multirow[t]{2}{*}{$g=8$} & $g_{7}^{2}$ & $g_{5}^{1}$ & $8 C_{(1)} * C_{(3)}+3 C_{(2)}^{* 2}=0$ & $C_{(1)} * C_{(3)}=C_{(2)}^{* 2}=0$ \\
\hline & $g_{6}^{2}$ & $g_{4}^{1}$ & $C_{(1)} * C_{(2)}=0$ & $\begin{aligned} C_{(0)} * C_{(1)} * C_{(2)} & =0 \\
C_{(1)}^{* 3} & =0\end{aligned}$ \\
\hline \multirow[t]{2}{*}{$g=9$} & $g_{7}^{2}$ & $g_{5}^{1}$ & $8 C_{(1)} * C_{(3)}+3 C_{(2)}^{* 2}=0$ & $\begin{aligned} C_{(1)} * C_{(3)}=C_{(2)}^{* 2} & =0 \\
C_{(0)} * C_{(1)} * C_{(3)}=C_{(0)} * C_{(2)}^{* 2} & =0 \\
C_{(1)}^{* 2} * C_{(2)} & =0\end{aligned}$ \\
\hline & $g_{6}^{2}$ & $g_{4}^{1}$ & $C_{(1)} * C_{(2)}=0$ & $\begin{aligned} C_{(0)} * C_{(1)} * C_{(2)} & =0 \\
C_{(0)}^{* 2} * C_{(1)} * C_{(2)} & =0 \\
C_{(0)} * C_{(1)}^{* 3} & =0\end{aligned}$ \\
\hline
\end{tabular}

with coefficients $Q_{i} \in \mathbb{Q}[r, d, g]$ that both $A$ and $B$ satisfy. We conclude because the first terms are the same: $A(1, d, g)=B(1, d, g)$ and $A(2, d, g)=B(2, d, g)$. To learn more about Zeilberger's algorithm, one can consult [PWZ96]. One can also find an explanation of the ZW package in [PS95].

\section{New relations in the tautological ring for nets and webs}

We have seen in the last section that the relations $C_{(i)}=0$ we can deduce from Theorem 1 could also be deduced from the theorem of Colombo and van Geemen. Do we obtain new relations modulo algebraic equivalence? Yes, we give the new relations for plane and space curves of low genus in Tables 1 and 2 .

The integer $A(2, d, g)=(d-1)(d-2) / 2-g$ is the well-known number of nodes for a plane curve of degree $d$ and genus $g$. So for every plane curve the integer $A(2, d, g)$ is positive. Conversely, when the integers $d$ and $g$ satisfy $g \geqslant 0$ and $(\sqrt{8 g+1}+3) / 2 \leqslant d$, there exists a plane curve with such invariants. As explained in the last section, when the curve is singular, one can consider the line passing through a singular point to obtain a $g_{d-2}^{1}$. Let us denote $d^{\prime}=d-2$ in this case. When the curve is smooth, it only admits a $g_{d-1}^{1}$. We denote $d^{\prime}=d-1$ in this case. We indicate in Table 1 the relations ${ }^{6}$ deduced from Theorem 1 . The new relations (those which cannot be deduced from $I_{g}$ and from Colombo and van Geemen's theorem applied to the $g_{d^{\prime}}^{1}$ the curve admits) ${ }^{7}$ are written in bold type.

Remark. For $g$ sufficiently large, one can always find $d$ such that a genus $g$ curve exists with a $g_{d}^{2}$ giving us new relations. Let us sketch the proof. The first non-trivial relation given by Theorem 1

\footnotetext{
${ }^{6}$ Keep in mind that Polishchuk used the cycles $p_{i+1}=\mathcal{F}\left(C_{(i)}\right)$ to describe $R$. So to translate the relations, one has to change $C_{(i)}$ into $p_{i+1}$ and the Pontryagin products into intersection products.

${ }^{7}$ More precisely, to define relations one should consider the kernel $\mathcal{R}$ of the map $\pi: \mathbb{C}\left[C_{(0)}, \ldots, C_{(g-1)}\right] \longrightarrow R$ which maps the indeterminate $C_{(i)}$ to the cycle $C_{(i)}$. One could also define (with Polishchuk's formula) a Fourier transform on $\mathbb{C}\left[C_{(0)}, \ldots, C_{(g-1)}\right]$ compatible with $\mathcal{F}$. In these terms, a new relation is an element of $\mathcal{R}$ which is not in the smallest ideal containing $I_{g}, C_{\left(d^{\prime}-1\right)}$ and stable under Fourier transform.
} 


\section{F. Herbaut}

TABLE 2. Relations deduced from Theorem 1 for space curves of genus $g \leqslant 9$.

\begin{tabular}{|c|c|c|c|c|}
\hline Genus & $g_{d}^{3}$ & $\begin{array}{l}g_{d^{\prime}}^{1} \text { deduced } \\
\text { from } g_{d}^{3} \\
\end{array}$ & $\begin{array}{c}\text { Relations deduced from } g_{d}^{3} \\
\text { with Theorem } 1\end{array}$ & Consequences \\
\hline$g=6$ & $g_{7}^{3}$ & $g_{3}^{1}$ & $C_{(0)} * C_{(1)}^{* 2}=0$ & \\
\hline$g=7$ & $g_{8}^{3}$ & $g_{4}^{1}$ & $9 C_{(0)} * C_{(1)} * C_{(2)}+C_{(1)}^{* 3}=0$ & \\
\hline$g=8$ & $g_{8}^{3}$ & $g_{4}^{1}$ & $9 C_{(0)} * C_{(1)} * C_{(2)}+C_{(1)}^{* 3}=0$ & $\begin{array}{r}C_{(1)} * C_{(2)}=0 \\
C_{(1)}^{* 3}=C_{(0)} * C_{(1)} * C_{(2)}=0\end{array}$ \\
\hline$g=9$ & $g_{9}^{3}$ & $g_{5}^{1}$ & $\begin{array}{r}8 C_{(0)} * C_{(1)} * C_{(3)} \\
+3 C_{(0)} * C_{(2)}^{* 2} \\
+2 C_{(1)}^{* 2} * C_{(2)}=0\end{array}$ & $\begin{aligned} C_{(1)} * C_{(3)}=C_{(2)}^{* 2} & =0 \\
C_{(1)}^{* 2} * C_{(2)} & =0 \\
C_{(0)} * C_{(2)}^{* 2} & =0 \\
C_{(0)} * C_{(1)} * C_{(3)} & =0\end{aligned}$ \\
\hline & $g_{8}^{3}$ & $g_{4}^{1}$ & $9 C_{(0)} * C_{(1)} * C_{(2)}+C_{(1)}^{* 3}=0$ & $\begin{array}{r}C_{(0)}^{* m} * C_{(1)} * C_{(2)}=0 \\
\text { for } m \in\{0 \ldots 2\} \\
C_{(0)}^{* n} * C_{(1)}^{* 3}=0 \\
\text { for } n \in\{0,1\}\end{array}$ \\
\hline
\end{tabular}

is

$$
\sum_{\substack{1 \leqslant a, b \\ a+b=d-3}}(a+1) !(b+1) ! C_{(a)} * C_{(b)}=0 \text { in } R_{(d-3)}^{g-2} .
$$

The relations of $I_{g}$ correspond to subspaces $R_{(i)}^{d+i}$ for $0 \leqslant d \leqslant g / 2-1$ and $d+1 \leqslant i \leqslant g-d-1$ (one has to see how the relations of the Theorem 0.1 of [Pol05] are parametrized). So when the inequality $d<g / 2+2$ holds, there is no relation of $I_{g}$ which belongs to $R_{(d-3)}^{g-2}$. Finally, if $d^{\prime}=d-1$, there is no relation deduced from the existence of the $g_{d^{\prime}}^{1}$ in $R_{(d-3)}^{g-2}$. If $d^{\prime}=d-2$, the only relation we can deduce from the $g_{d^{\prime}}^{1}$ in $R_{(d-3)}^{g-2}$ is $C_{(0)} * C_{(d-3)}=0$ which is different from (17).

The integer $A(3, d, g)$ is the number of quadrisecants to the space curve $\Phi(C)$. Let us recall Cayley's formula

$$
A(3, d, g)=\frac{(d-2)(d-3)^{2}(d-4)}{12}-\frac{g\left(d^{2}-7 d+13-g\right)}{2} .
$$

When $A(3, d, g)$ is non-zero, the curve admits a $g_{d-4}^{1}$ and so $C_{(d-5)}$ is zero modulo algebraic equivalence. We note $d^{\prime}=d-4$ in this case. Otherwise, we deduce from the existence of a $g_{d-3}^{1}$ the vanishing of $C_{(d-4)}$. We note $d^{\prime}=d-3$. We give in Table 2 the results we obtain by Theorem 1 for space curves of low genus. As above, a new relation is written in bold type. It means the relation cannot be deduced from the theorem of Colombo and van Geemen applied to the $g_{d^{\prime}}^{1}$ and from the relations of $I_{g}$. With the same argument as in the case of a $g_{d}^{2}$, one can show that for $g$ sufficiently large there exists a genus $g$ space curve for which Theorem 1 gives us new relations.

\section{ACKNOWLEDGEMENTS}

This paper is part of my thesis written at the University of Nice Sophia-Antipolis, in the Laboratoire Jean Dieudonné. I would like to thank my PhD advisor, Professor Arnaud Beauville, for his guidance and his constant support. I am grateful to Claire Voisin and to the anonymous referee for much 


\section{Algebraic CyCles on the Jacobian of A CURve}

useful advice. It is also a pleasure to thank George Hitching for his help with the English. Finally, I would like to thank Claire Voisin for the proof of Proposition 2.

\section{REFERENCES}

ACGH85 E. Arbarello, M. Cornalba, P. Griffiths and J. Harris, Geometry of algebraic curves (Springer, Berlin, 1985).

Bea83 A. Beauville, Quelques remarques sur la transformation de Fourier dans l'anneau de Chow d'une variété abélienne, in Algebraic geometry, Tokyo-Kyoto, 1982, Lecture Notes in Mathematics, vol. 1016 (Springer, Berlin, 1983), 238-260.

Bea86 A. Beauville, Sur l'anneau de Chow d'une variété abélienne, Math. Ann. 273 (1986), 647-651.

Bea04 A. Beauville, Algebraic cycles on Jacobian varieties, Compositio Math. 140 (2004), 683-688.

Cer83 G. Ceresa, $C$ is not algebraically equivalent to $C^{-}$in its Jacobian, Ann. of Math. (2) 117 (1983), $285-291$.

CV93 E. Colombo and B. van Geemen, Note on curves in a Jacobian, Compositio Math. 88 (1993), 333-353.

ELMS89 D. Eisenbud, H. Lange, G. Martens and F.-O. Schreyer, The Clifford dimension of a projective curve, Compositio Math. 72 (1989), 173-204.

Fak96 N. Fakhruddin, Algebraic cycles on generic Abelian varieties, Compositio Math. 100 (1996), $101-119$.

Ful83 W. Fulton, Intersection theory (Springer, Berlin, 1983).

GKP89 R. Graham, D. Knuth and O. Patashnik, Concrete mathematics - a foundation for computer science (Addison-Wesley, Reading, MA, 1989).

Ike03 A. Ikeda, Algebraic cycles and infinitesimal invariants on Jacobian varieties, J. Algebraic Geom. 12 (2003), 573-603.

Iza05 E. Izadi, Deforming curves in Jacobians to nonJacobians I: curves in $C^{(2)}$, Geom. Dedicata 116 (2005), 87-109.

Kou93 A. Kouvidakis, Divisors on symmetric products of curves, Trans. Amer. Math. Soc. 337 (1993), $117-128$.

Mar05 G. Marini, Tautological cycles on Jacobian varieties, Preprint (2005), arXiv:math/0509659.

Pol05 A. Polishchuk, Universal algebraic equivalences between tautological cycles on Jacobians of curves, Math. Z. 251 (2005), 875-897.

PS95 P. Paule and M. Schorn, A Mathematica version of Zeilberger's algorithm for proving binomial coefficient identities, J. Symbolic Comput. 20 (1995), 673-698.

PWZ96 M. Petkovsek, H. Wilf and D. Zeilberger, $A=B$ (AK Peters, Wellesley, MA, 1996).

VK07 G. van der Geer and A. Kouvidakis, Cycle relations on Jacobian varieties. With an appendix by Don Zagier, Compositio Math. 143 (2007), 900-908.

Fabien Herbaut herbaut@math.unice.fr

Laboratoire J. A. Dieudonné (UMR 6621 du CNRS), Université de Nice Sophia-Antipolis, Parc Valrose, 06108 Nice cedex 2, France 